\title{
Medical genetics in China: a western view
}

The development of medical genetics services in a country of more than 1000 million people, with limited financial and scientific resources, and in conjunction with a strictly applied policy of family limitation, presents challenges and problems that are perhaps unique in the world at the present time. An opportunity to see this situation at first hand came recently through a visit of five clinical geneticists from the United Kingdom* to Beijing (Peking), Shanghai, and, very briefly, Guangzhou (Canton). Although the inevitable limitations of time and geography allowed only a fragmentary glimpse of the whole picture, the members of the group all found the experience a fascinating one and something worthy of being recorded. It was also an experience that challenged some of the principles that underly the practice of medical genetics in most European countries and North America. Gaining a clear view of the situation was greatly helped by the open and hospitable approach of the scientists and clinicians whom we met, who included not only geneticists, but paediatricians, obstetricians, and family planning experts.

This is a brief account of some of the most interesting aspects. A future issue of Journal of Medical Genetics will include a series of papers by Chinese authors on some specific topics, in particular the prevalence and distribution of major genetic disorders, while some more detailed aspects of the visit will appear in a forthcoming issue of Biology and Society. Readers may also like to refer back to the article by W F Bodmer and C A Clarke written after their visit ( $J$ Med Genet 1979;16:330-7).

THE PATTER N OF GENETIC DISEASE

In a country that is a sub-continent, with extremes of climate and terrain, and with numerous minority populations, any generalisations must be dangerous. The haemoglobinopathies form a clear and well documented example of this, with a low incidence of the thalassaemias and haemoglobin variants in Beijing, increasing to the south, with an incidence of $15 \%$ for $\alpha$ thalassaemia in the south-west border province of Guangxi. Phenylketonuria is as common as in Western Europe ( 1 in 15000$)$, but both galactosaemia and cystic fibrosis are rare, while $\alpha_{1}$ antitrypsin deficiency is not seen, variants being without harmful effects. Wilson's disease is common in both Beijing and Shanghai. Among the other Mendelian disorders, the best documented were inherited eye disorders, with a thorough study of 16 different conditions based on the Shanghai Population Genetics Research Group. This study has also provided valuable data on local recurrence risks for use in genetic counselling for polygenic eye disorders and for congenital deafness. Duchenne muscular dystrophy and the haemophilias are frequent clinical problems, but precise figures were not available. However, the frequency of Down's syndrome was 1 to 1.5 per 1000 .

Information on common malformations proved much less easy to obtain. Several small scale series had been done or were in progress in both centres, but no satisfactory major study existed; it was not always clear whether stillbirths had been included. Neural tube defects were certainly not rare, with a probable frequency of around 1.0 per 1000; an incidence of 1.7 per 1000 was quoted for a study in Sichuan province. There seemed to be no separate recognition of dysmorphology and it was difficult to obtain information on the frequency and type of specific syndromes. 
MEDICAL GENETICS SERVICES

Medical genetics in China is a comparatively recent development, the influence of Lysenko and then the upheavals of the Cultural Revolution having effectively prevented any widespread work in this field until around 1976. Since then it is clear that rapid progress has been made in a number of fields, though there does not yet seem to have been any uniform planning of laboratory or clinical genetics developments on a national level. There are more than 400 members of the Section of Human and Medical Genetics of the Chinese Genetics Society and also a separate Chinese Eugenics Society. As in Britain at an earlier period, new facilities have developed around experienced people who already had a specific interest in the field, mostly medically trained, but some with a basic science background.

The major cytogenetics units we visited had a high quality of chromosome preparations, their work being orientated to prenatal diagnosis and in particular the analysis of chorion villus biopsies, the direct technique of cytogenetic analysis being used. Screening for inherited metabolic diseases was in progress, but only in Shanghai was dietary treatment available for phenylketonuria. In neither Beijing nor Shanghai was there any systematic heterozygote screening for haemoglobinopathies, but neither shows the high incidence of these seen in south-west China.

All the units showed a particular interest in the development of molecular genetics techniques and an awareness of the likely value in prenatal diagnosis. Several had sent workers abroad specifically to set up DNA techniques and in Shanghai Children's Hospital DNA analysis in the thalassaemias and PKU was already in regular use for prenatal diagnosis and in research. The funding of this work seems largely to depend on individual initiative, with the possibility of government research grants and, in some cases, funding from abroad. There has not been any special allocation of funds so far to develop the service related aspects of the work, whether molecular or cytogenetic, and the number of such samples dealt with is small.

Turning to slinical genetics services, the provision of systematic genetic counselling still seemed to be at an early stage of development. Most of those full time in the field trained originally in paediatrics or general internal medicine; obstetricians are also much involved in genetic counselling as a result of the specialty's close relationship to decisions on prenatal diagnosis, termination of pregnancy, and contraception. There seemed to be a considerable difference between the genetic counselling given by specialists, whose approach was in many ways similar to that seen in Britain, and that given by obstetric and family planning personnel, where the approach appeared more directive, with emphasis on termination if there was any doubt as to the genetic risks. The majority of clinicians, however, told us that at present the final decision was left to the couple. Both chorion biopsy and ultrasound appeared to be well developed and widely used in the Beijing and Shanghai University Obstetrics Departments, one unit reporting as many chorion biopsies as amniocenteses. With almost all urban births in hospital and early booking the rule, the further extension and organisation of these services should not pose major difficulties.

GENETIC COUNSELLING AND THE 'ONE CHILD FAMILY'

For any visitor from Britain the impact of China's population problem is immediate and compelling. The general view expressed, widely and frankly, was simply that "there are too many of us", and that failure to take firm action now would inevitably lead to disastrous consequences later. According to the State Family Planning Commission, the steep fall in infant mortality (down to 35 per 1000 now from 200 before 1949) together with the increased longevity (now 68 compared with 35 before 1949) means that the population will inevitably increase from its present level of over 1000 million, however strict is the limitation of family size.

The policy of one child per family is primarily promoted in the cities by massive publicity, readily available contraception (mainly IUD), and abortion, and by strong social and workplace pressures on the individual couple, even though there is currently no legal prohibition of a second child. It was made quite clear that permission was required before conception and that a second 
child would be allowed only in exceptional circumstances. Data from Beijing showed a mean family size of $1 \cdot 1$, suggesting that a single child is indeed the strictly enforced rule, although in rural and minority areas a less strict policy appears to operate.

Since the occurrence of an abnormality in the first child is one of the reasons for a second child sometimes being allowed, the role of genetic counselling and of those giving it is in this respect radically different from that in Western societies. Many referrals were after the birth of an abnormal child to determine whether recurrence was likely, in which case it was clear that permission for a second child would not be given, unless prenatal diagnosis was possible. The genetic counsellor is thus inevitably in the position of arbiter, since on his estimate of risk will depend the decision on that couple's reproductive future. Sometimes this may work to the couple's advantage; thus, in one clinic, the mother of an achondroplastic child (due to a mutation) was undoubtedly going to be permitted a second child, which might not have been the case had the diagnosis and low risk not been clearly identified. In other situations the reverse might have happened.

It was difficult to establish the extent to which the professionals and other people in the community were able to influence the decision, and also what constituted a high risk, while complexities of severity and lethality did not appear always to influence the situation greatly. Not surprisingly, different people expressed varying views, but regardless of this it seemed that the onus was on a couple with an abnormal child to prove that the recurrence risk would be low before a further child could be considered.

For anyone whose practice of genetic counselling is founded on non-directive principles, this situation is both unfamiliar and disturbing. The responsibility for giving information and advice that may radically alter peoples' lives weighs heavily on one, even when the final decision is left to the people concerned; even those favouring a 'directive' approach accept that people have the right to ignore their advice. However, in the context of the one child family it would be easy to see restriction on the couple with an abnormal child as logical, given the general requirement for permission before conception. Thus, the differences in approach to medical genetics appear to be founded more on the general relationship of people to society in reproductive decision making, rather than on any specific difference in the role of genetic counselling.

EUGENICS IN CHINA

The term 'eugenics' produces unease in clinical geneticists in Europe and North America, at least in part for historical reasons, with the shadow of Nazi Germany and America of the 1930s still uncomfortably close. The emphasis of clinical genetics has been on individual problems rather than on attempts to alter the genetic structure of populations. Despite this unease, the problem has never really gone away and many of the undoubted successes of medical genetics have been in the field of population screening for abnormalities, whether prenatal (as for neural tube defects, Down's syndrome, and thalassaemia) or postnatal (as for phenylketonuria). The development of molecular genetics, with the potential for identifying genetic susceptibility, and for prediction of such disorders as Huntington's chorea, as well as the success of in vitro fertilisation, has again brought concern for the ethical implications of these new techniques.

The word 'eugenics' is used more widely in China than currently in the West, and it is difficult to be certain of its precise meaning; indeed this probably varies with its context. Undoubtedly much of what is termed eugenics in China would be included under what we would call genetic counselling, genetic screening, or clinical genetics. Equally clear, at least among clinicians and geneticists in China, as in the UK, was the view that eugenics was not concerned with attempts positively to improve the human race or to eliminate the genetically defective. The most general view seemed to be that eugenics implied processes designed to ensure that those children who were to be born were as far as possible 'normal'. How to achieve this, in the context of strict family limitation, emerged as the most significant difference in the approach to medical genetics between 
China and the West, a difference revolving around the current debate in China about the need for 'eugenic laws'.

In Europe and America, such laws as have at times existed forbidding marriage and reproduction of certain persons with genetic disorders have almost universally been repealed (apart from those concerning incest); Japan and perhaps South Korea remain as exceptions. These laws have widely been regarded as discriminatory and ineffective, as well as being based in some cases on ill defined criteria with no valid genetic basis.

Following the only recent opening of China to Western concepts of genetics, discussions are in progress that could lead China to the introduction of laws for eugenic purposes, regulating marriage and childbearing. On a short visit to a completely unfamiliar country and society, in which we were able to glimpse only small fragments of the issues, attitudes, and problems involved, it may be presumptuous to give unsolicited advice on proposed eugenic laws. But equally, when invited to discuss the subject informally with those involved in politics and decision making, one was able to make clear one's personal views. This was particularly important since the subject was still one that was far from decided, at least in extent and nature. It seemed that the opinion of visitors would be listened to and considered carefully, and that there was a strong desire for contact with and information, both general and technical, from Western experts.

The main conclusion that could be reached on this difficult and sensitive topic was that the principal aims underlying any eugenic laws would all be more effectively achieved by other means. More tangible results in terms of prevention of genetic disease would come from the development of medical genetics services, including genetic counselling, new advances in prenatal diagnosis, and population screening programmes for specific major problems. Not only did it seem likely that any law would be inevitably inflexible and rapidly outdated, but there would be serious difficulties in defining groups to be included. Prohibition applied to those known to have a family history of genetic disease would do little to reduce the general frequency, and disorders currently beingo studied might be hidden by the families for fear of restriction being imposed. It also seemed distinctly possible that the development of medical genetics might be held back by being associated with such laws.

The Chinese have exhibited a very responsible attitude to population problems and have adopted radical measures for their control, but to link these with eugenic laws would undoubtedly risk the alienation of Western medical geneticists, as there would be widespread reluctance among both scientists and clinicians to be associated with such a development. It is profoundly to be hoped that medical genetics in China is able to develop in a way that not only allows benefit to be brought to its people, but also promotes the warm and lively international understanding that made this visit enjoyable, stimulating, challenging, and in all respects worthwhile.

Peter S HARPer AND RodNey HARRIS 